\title{
Particle Production from Soft Processes
}

\author{
Patricia Fachini \\ Brookhaven National Laboratory, Upton, NY 11973 USA
}

Received on 30 October, 2006; revised version received on 21 March, 2007

\begin{abstract}
Relativistic heavy-ion collisions provide a unique environment to study matter under extreme conditions of high temperature and energy density. In the soft $p_{\mathrm{T}}$ region $(\leq 2 \mathrm{GeV} / c)$ particle production is governed by the bulk properties (e.g. hydrodynamic pressure, freeze-out temperature) of the collisions. Total particle yields as well as their distributions in phase space are strongly dependent on these properties. These bulk properties will be discussed and we will compare our heavy-ion results to measurements in the $p+p$ reference system, where multiparticle processes such as recombination and rescattering are generally thought to be negligible. However, we will show evidence for $\pi^{+} \pi^{-}$rescattering interactions even in $p+p$ collisions.
\end{abstract}

Keywords: Soft interaction; Elliptic flow; Mass modification

\section{ELLIPTIC FLOW}

In non-central collisions the initial spatial anisotropy is transformed into an anisotropy in momentum-space if sufficient interactions occur among the constituents within the system. Once the system has expanded enough to quench the spatial anisotropy, further development of momentum anisotropy ceases. This self-quenching process happens quickly, so elliptic flow is primarily sensitive to the early stages of the collisions [1].

\section{A. Hydrodynamics}

The elliptic flow $v_{2}$ as a function of $p_{\mathrm{T}}$ for $K_{S}^{0}, \Lambda, \phi, \Xi$, and $\Omega$ is depicted in Fig. $1[2,3]$. The $\phi, \Xi$, and $\Omega$ have low hadronic cross-sections, therefore the large $v_{2}$ observed suggest that the elliptic flow is built up in the partonic stage. The expected range of $v_{2}$ from hydrodynamic calculations is also shown in Fig. 1.

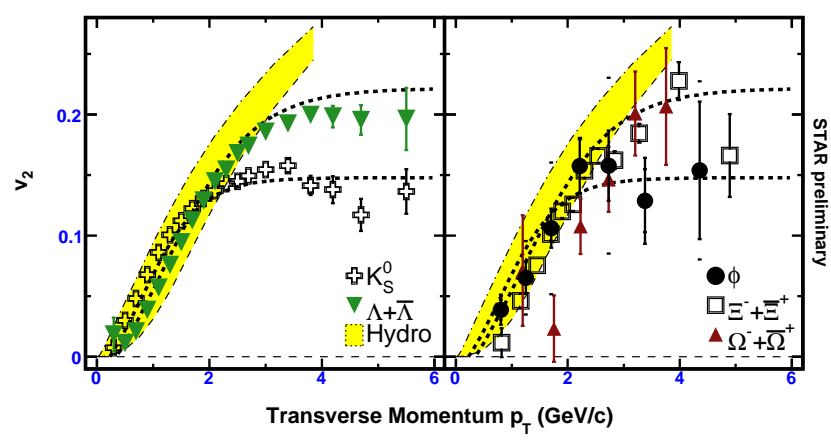

FIG. 1: Azimuthal anisotropy $v_{2}$ for strange (left panel) and multistrange (right panel) hadrons in minimum bias $\mathrm{Au}+\mathrm{Au}$ collisions [2]. Data measured by STAR. The dashed lines show a common fit to the $K_{S}^{0}$ and $\Lambda+\bar{\Lambda}$ data [5]. The shaded areas are hydrodynamic calculations [1]

A more detailed comparison can be seen in Fig. 2, where the mass dependent hydrodynamic results [1] are compared to the $v_{2}$ measurements of $\pi, K, p$, and $\Lambda$ [4]. Hydrodynamics describes well the mass dependence observed in the data that is characteristic of a common flow velocity. Since an ideal hydrodynamic fluid is a thermalized system with a zero mean free path that yields the maximum possible $v_{2}$, the good agreement between the measured $v_{2}$ and the hydro results [1] suggests thermalization in heavy-ion collisions at RHIC.

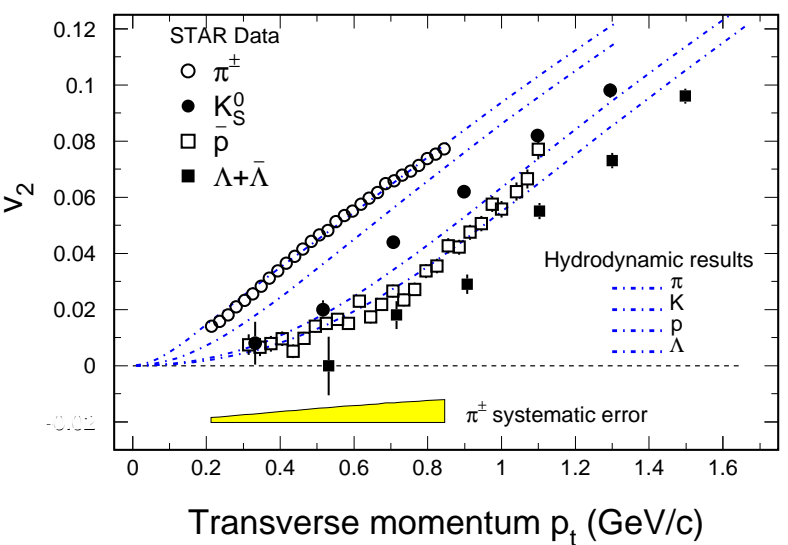

FIG. 2: $v_{2}$ as a function of $p_{\mathrm{T}}$ from minimum bias $\mathrm{Au}+\mathrm{Au}$ collisions [4] measured by STAR. The dotted-dashed lines are hydrodynamic calculations using an equation of estate (EOS) with a firstorder hadron quark-gluon plasma phase transition [1]. The description of the data worsen if a hadronic EOS is used [1].

\section{B. Constituent Quark Scaling}

While hydrodynamic calculations keep increasing as a function of $p_{\mathrm{T}}$, the measured $v_{2}$ saturates at $p_{\mathrm{T}}>2 \mathrm{GeV} / c$ [2]. The saturation value for mesons is about $2 / 3$ of that for baryons. This separation pattern holds for $\pi, K, \Lambda$, and $\Xi$, and seems to hold for $\phi$ and $\Omega[3,6]$. This result and the baryonmeson splitting of the high $p_{\mathrm{T}}$ suppression pattern [7] suggest the relevance of the constituent quark degrees of freedom in the intermediate $p_{\mathrm{T}}$ region [8]. $v_{2}$ scaled by the number of valence quarks $n$ as a function of $p_{\mathrm{T}} / n$ is depicted in Fig. 3. The lower panel of Fig. 3 displays the ratio between the measurements and a polynomial fit to all the data. At low $p_{\mathrm{T}} / n$ 


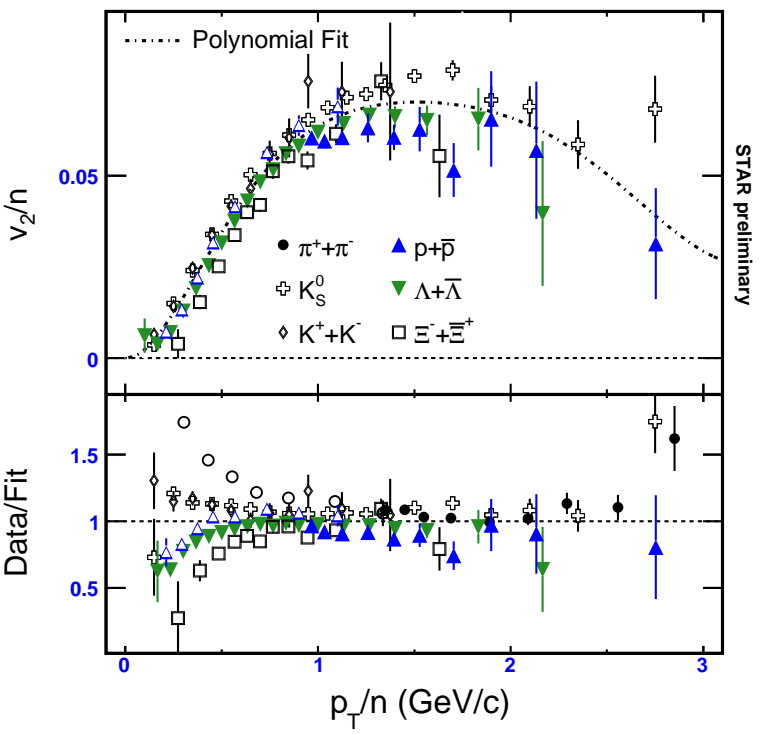

FIG. 3: Measurements by STAR of the scaled $v_{2}\left(p_{\mathrm{T}} / n\right) / n$ for identified hadrons (upper panel) and the ratio between the measurements and a polynomial fit through all data points (lower panel) except the pions for $\sqrt{s_{\mathrm{NN}}}=200 \mathrm{GeV}$ minimum bias $\mathrm{Au}+\mathrm{Au}$ collisions [2].

the observed deviations from the fit follow a mass ordering which is expected from hydrodynamics. At higher $p_{\mathrm{T}}$, all $v_{2} / n$ measurements are reasonably close to unity showing the constituent quark scaling.

\section{FREEZE-OUT PROPERTIES}

The measured particle spectra and yields [9] and event-byevent $\left\langle p_{\mathrm{T}}\right\rangle$ fluctuations [10] indicate a nearly chemically and kinetically equilibrated system at the final freeze-out stage.

\section{A. Chemical and Kinetic Freeze-out Parameters}

STAR has now measured hadron distributions at $\sqrt{s_{\mathrm{NN}}}=$ 200 and $62 \mathrm{GeV}[11,12]$. Chemical freeze-out properties were extracted from stable particle ratios within the thermal model [28]. Kinetic freeze-out properties from particle $p_{\mathrm{T}}$ distributions were extracted within the blast wave model [14]. The extracted chemical freeze-out temperature $T_{\mathrm{ch}}$, the strangeness suppression factor $\gamma_{s}$, kinetic freeze-out temperature $T_{\text {kin }}$ and the average radial flow velocity $\left\langle\beta_{\mathrm{T}}\right\rangle$ at $\sqrt{s_{\mathrm{NN}}}$ $=62 \mathrm{GeV}$ are found to be qualitatively the same as those obtained at $\sqrt{s_{\mathrm{NN}}}=200 \mathrm{GeV}$ and resonance decays are found to have no significant effect on the extract kinetic freeze-out parameters [12].

$T_{\mathrm{ch}}$ is independent of centrality. $T_{\mathrm{kin}}$ obtained from $\pi, K$, and $p$ decreases as a function of centrality, while the corresponding $\left\langle\beta_{\mathrm{T}}\right\rangle$ increases. This is evidence that the system expands between chemical and kinetic freeze-outs, which brings the system to a lower temperature. $\gamma_{s}$ increases from $p+p$ to peripheral and central $\mathrm{Au}+\mathrm{Au}$ collisions. In central $\mathrm{Au}+\mathrm{Au}$ collisions, $\gamma_{s}$ is $\sim 1$ suggesting that strangeness is saturated. The $T_{\mathrm{ch}}$ of $\phi, \Xi$, and $\Omega$ is higher than that of $\pi, K$, and $p$, while the $\left\langle\beta_{\mathrm{T}}\right\rangle$ is lower. Noting that the $\phi, \Xi$, and $\Omega$ have small hadronic cross-sections, they may chemically and kinetically freeze-out at the same time.

\section{B. Resonances}

In order to study different hadronic interactions effects on different resonances in relativistic heavy-ion collisions, one should compare the potential differences in the ratio of the resonance to its corresponding ground state. Figure 4 depicts the $K^{*} / K^{-}, \phi / K^{-}$, and $\rho^{0} / \pi^{-}$yield ratios as a functions of $d N_{r m c h} / d \eta$ for $\mathrm{Au}+\mathrm{Au}$ collisions at $\sqrt{s_{\mathrm{NN}}}=200 \mathrm{GeV}$ measured by STAR [34]. All yield ratios have been normalized to the corresponding yield ratio measured in minimum bias $p+p$ collisions at the same c.m. system energy and indicated by the solid line in Fig. 4. The $K^{*} / K^{-}$yield ratio for central $\mathrm{Au}+\mathrm{Au}$ collisions is significantly lower than the minimum bias $p+p$ measurement at the same $\sqrt{s_{\mathrm{NN}}}$.

In addition, the statistical model prediction of $K^{* 0} / K^{-}$is $2 \sigma$ higher than the measurement [34]. The lower $K^{*} / K^{-}$yield ratio measured may be due to the rescattering of the $K^{* 0}$ decay products. Due to the relatively long lifetime of the $\phi$ meson and the negligible $K K$ cross-section, the rescattering of the $\phi$ decay products and the phi regeneration should be negligible. The statistical model prediction for the $\phi / K^{-}$yield ratio indeed agrees with the measurement [34].

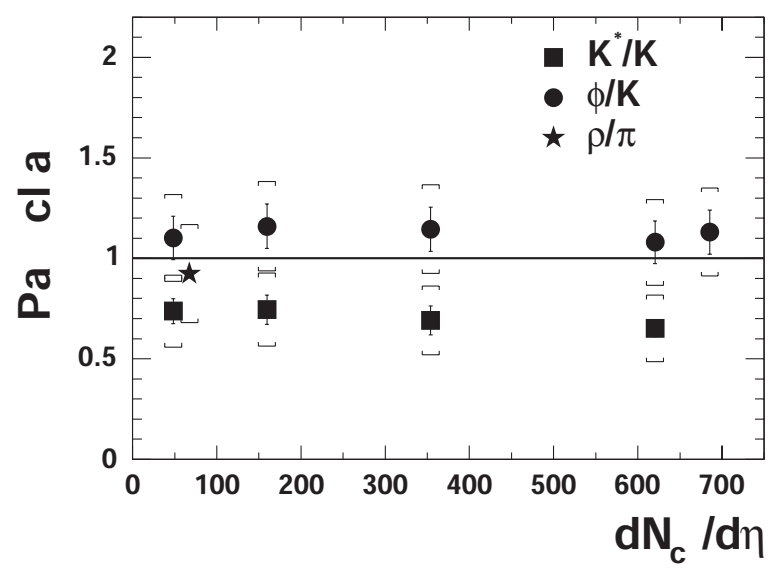

FIG. 4: The $K^{*} / K^{-}, \phi / K^{-}$, and $\rho^{0} / \pi^{-}$yield ratios as a functions of $d N_{\mathrm{ch}} / d \eta$ for $\mathrm{Au}+\mathrm{Au}$ collisions at $\sqrt{s_{\mathrm{NN}}}=200 \mathrm{GeV}$ measured by STAR [34]. All yield ratios have been normalized to the corresponding yield ratio measured in minimum bias $p+p$ collisions at the same c.m. system energy and indicated by the solid line. Both statistical and systematical uncertainties are shown. 


\section{EVIDENCE FOR $\pi^{+} \pi^{-}$SCATTERING IN $p+p$ COLLISIONS}

The $\rho^{0}$ was measured via its hadronic decay channel in minimum bias $p+p$ and $\mathrm{Au}+\mathrm{Au}$ collisions at RHIC and a mass shift of -40 and $-70 \mathrm{MeV} / c^{2}$ of the position of the $\rho^{0}$ was observed, respectively. While no explanations were explicitly attributed to the mass shift in $p+p$ collisions, the possible explanations for the apparent modification of the $\rho^{0}$ meson properties in $\mathrm{Au}+\mathrm{Au}$ collisions were attributed to dynamical interactions with the surrounding matter, interference between various $\pi^{+} \pi^{-}$scattering channels, phase space distortions due to the rescattering of pions forming $\rho^{0}$ and Bose-Einstein correlations between $\rho^{0}$ decay daughters and pions in the surrounding matter [16].

The $\rho^{0}$ meson measured in the dilepton channel probes all stages of the system formed in relativistic heavy-ion collisions because the dileptons have negligible final state interactions with the hadronic environment. Heavy-ion experiments at CERN show an enhanced dilepton production cross section in the invariant mass range of $200-600 \mathrm{MeV} / c^{2}$, showing that the $\rho^{0}$ is broadened rather than shifted $[17,18]$.

The modification of the $\rho^{0}$ properties in heavy-ion collisions has been expected [19], contrary to the modifications of the $\rho^{0}$ properties in $p+p$ collisions. Similar modifications of that measured in $p+p$ collisions at RHIC has been observed before at CERN-LEBC-EHS and CERN-LEP. Until now, the mass shift measured in $p+p$ at CERN-LEBC-EHS has been attributed to phase space.

\section{A. Discussion $\rho$ mass average from the Particle Data Group} (PDG)

We will first discuss the $\rho$ mass average from the PDG [26]. The $\rho^{0}$ mass average $775.8 \pm 0.5 \mathrm{MeV} / c^{2}$ from $e^{+} e^{-}$was obtained from either $e^{+} e^{-} \rightarrow \pi^{+} \pi^{-}$or $e^{+} e^{-} \rightarrow \pi^{+} \pi^{-} \pi^{0}$. This means that the $\rho^{0}$ mass average was obtained from exclusive leptonic reactions. Similarly, the $\rho^{ \pm}$mass average $775.5 \pm$ $0.5 \mathrm{MeV} / \mathrm{c}^{2}$ was also was obtained from exclusive leptonic reactions.

The $\rho$ averages reported by the PDG from reactions other than leptonic interactions are systematic lower than the value obtained from leptonic exclusive interactions by $\sim 10 \mathrm{MeV} / c^{2}$ [26]. The $\rho$ production in these hadronic reactions are inclusive and exclusive. In the case of inclusive productions, the phase space was take into account when the $\rho$ mass was measured (e.g. [20]).

These observations lead us to conclude that the $\rho$ mass depends on specific interactions, e.g. whether the $\rho$ is produced in inclusive or exclusive reactions. Since a leptonic reaction and exclusive measurement of the $\rho^{0}$ lead to a negligible modification of any kind of the $\rho^{0}$ mass, the average $775.8 \pm 0.5$ $\mathrm{MeV} / c^{2}[26]$ from $e^{+} e^{-}$should correspond to the $\rho^{0}$ mass in the vacuum.

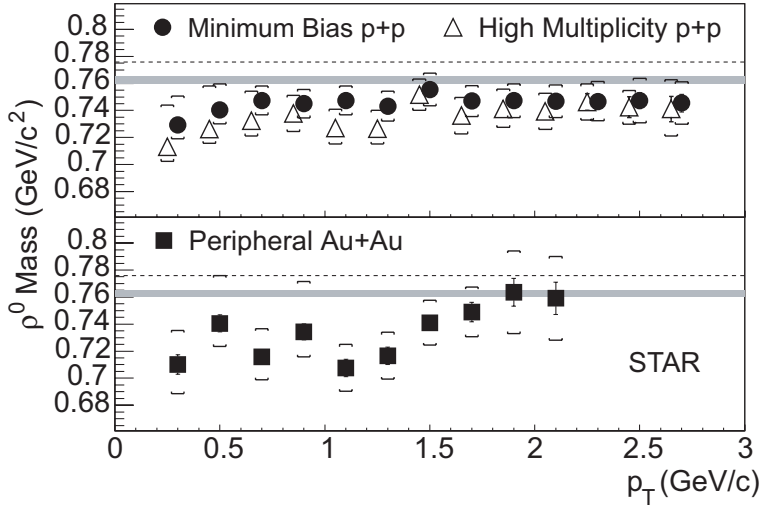

FIG. 5: The $\rho^{0}$ mass as a function of $p_{\mathrm{T}}$ for minimum bias $p+p$ (filled circles), high multiplicity $p+p$ (open triangles), and peripheral $\mathrm{Au}+\mathrm{Au}$ (filled squares) collisions measured by STAR. The error bars indicate the systematic uncertainty. Statistical errors are negligible. The $\rho^{0}$ mass was obtained by fitting the data to the $\mathrm{BW} \times \mathrm{PS}$ functional form described in [16]. The dashed lines represent the average of the $\rho^{0}$ mass measured in $e^{+} e^{-}$[26]. The shaded areas indicate the $\rho^{0}$ mass measured in $p+p$ collisions [20]. The open triangles have been shifted downward on the abscissa by $50 \mathrm{MeV} / \mathrm{c}$ for clarity.

\section{B. $\rho^{0}$ mass shifts at RHIC, CERN-LEBC-EHS, and CERN-LEP}

The $\rho^{0}$ was measured in the hadronic decay channel $\rho^{0} \rightarrow$ $\pi^{+} \pi^{-}$at RHIC, CERN-LEBC-EHS, and CERN-LEP in inclusive production. At RHIC, the STAR collaboration measured the $\rho^{0}$ at $\sqrt{s_{\mathrm{NN}}}=200 \mathrm{GeV}$ at midrapidity $(|y|<0.5)$ and observed mass shifts of the position of the $\rho^{0}$ peak of about $-40 \mathrm{MeV} / c^{2}$ and $-70 \mathrm{MeV} / c^{2}$ in minimum bias $p+p$ and peripheral $\mathrm{Au}+\mathrm{Au}$ collisions, respectively [16]. The $\rho^{0}$ mass obtained from the $\mathrm{BW} \times \mathrm{PS}$ fit to the invariant mass distributions after the subtraction of the like-sign background is depicted in Fig. 5, where it is clear that the phase space does not account for the measured mass shifts of the position of the $\rho^{0}$ peak.

At CERN-LEBC-EHS, NA27 measured the $\rho^{0}$ in minimum bias $p+p$ at $\sqrt{s}=27.5 \mathrm{GeV}$ for $x_{\mathrm{F}}>0$, where $x_{\mathrm{F}}$ is the ratio between the longitudinal momentum and the maximum momentum of the meson, and reported a mass of 762.6 $\pm 2.6 \mathrm{MeV} / \mathrm{c}^{2}[20]$. The $\rho^{0}$ signal can be seen even before background subtraction. The invariant $\pi^{+} \pi^{-}$mass distribution was fit to the $\mathrm{BW}(\mathrm{NA} 27) \times \mathrm{PS}(\mathrm{NA} 27)$ plus a background function [20]. In this analysis, the phase space function (PS(NA27)) used is the same as the combinatorial background (BG). The invariant $\pi^{+} \pi^{-}$mass distribution after subtraction of the mixed-event reference distribution is shown in Fig. 6. The vertical dashed line represent the average of the $\rho^{0}$ mass $775.8 \pm 0.5 \mathrm{MeV} / c^{2}$ measured in $e^{+} e^{-}$[26]. The vertical solid line is the $\rho^{0}$ mass $762.6 \pm 2.6 \mathrm{MeV} / c^{2}$ reported by NA27 [20]. As shown in Fig. 6, the position of the $\rho^{0}$ peak is shifted by $\sim 30 \mathrm{MeV} / c^{2}$ compared to the $\rho^{0}$ mass in the vacuum $775.8 \pm 0.5 \mathrm{MeV} / c^{2}[26]$. 


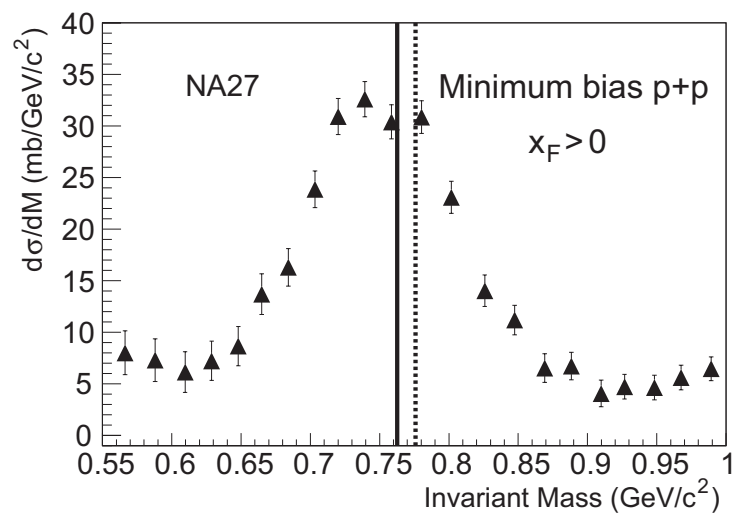

FIG. 6: The invariant $\pi^{+} \pi^{-}$mass distribution after background subtraction for minimum bias $p+p$ collisions measured by NA27. For details see [20]. The vertical dashed line represent the average of the $\rho^{0}$ mass $775.8 \pm 0.5 \mathrm{MeV} / c^{2}$ measured in $e^{+} e^{-}$[26]. The vertical solid line is the $\rho^{0}$ mass $762.6 \pm 2.6 \mathrm{MeV} / c^{2}$ reported by NA27 [20].

As mentioned, NA27 obtained the $\rho^{0}$ mass by fitting the invariant $\pi^{+} \pi^{-}$mass distribution to the $\mathrm{BW} \times \mathrm{PS}$ function, and they reported a mass of $762.6 \pm 2.6 \mathrm{MeV} / c^{2}$, which is $\sim 10$ $\mathrm{MeV} / c^{2}$ lower than the $\rho^{0}$ mass in the vacuum. Ideally, the PS factor should have accounted for the shift on the $\rho^{0}$ peak, and the mass obtained from the fit should have agreed with the $\rho^{0}$ mass in the vacuum. However, just like in the STAR measurement, this was not the case, since the phase space did not account for the mass shift on the position of the $\rho^{0}$ peak.

At CERN-LEP, OPAL, ALEPH and DELPHI measured the $\rho^{0}$ in inclusive $e^{+} e^{-}$reactions at $\sqrt{s}=90 \mathrm{GeV}$ [21-24]. Even though OPAL never reported the value of the $\rho^{0}$ mass, OPAL reported a shift on the position of the $\rho^{0}$ peak by $\sim 70 \mathrm{MeV} / c^{2}$ at low $x_{\mathrm{p}}$, where $x_{\mathrm{p}}$ is the ratio between the meson and the beam energies, and no shift at high $x_{\mathrm{p}}\left(x_{\mathrm{p}} \sim 1\right)$ [21, 22]. OPAL also reported a shift in the position of the $\rho^{ \pm}$peak from -10 to $-30 \mathrm{MeV} / c^{2}$, which was consistent with the $\rho^{0}$ measurement [23]. ALEPH reported the same shift on the position of $\rho^{0}$ peak observed by OPAL [24]. Both OPAL and ALEPH used the like-sign method to subtract the background. DELPHI fit the raw invariant $\pi^{+} \pi^{-}$mass distribution to the $(\mathrm{BW} \times \mathrm{PS}+$ BG) function for $x_{\mathrm{p}}>0.05$ and reported a $\rho^{0}$ mass of $757 \pm$ $2 \mathrm{MeV} / c^{2}$ [25], which is five standard deviations below the $\rho^{0}$ mass in the vacuum $\left(775.8 \pm 0.5 \mathrm{MeV} / c^{2}\right)$. As one can see, similarly to NA27, DELPHI assumed that the phase space was described by the background function. Bose-Einstein correlations were used to describe the shift on the position of $\rho^{0}$ peak. However; high (even unphysical) chaocity parameters $(\lambda \sim 2.5)$ were needed $[21,22,24]$.

\section{Phase space in $p+p$ collisions}

In $p+p$ collisions, most models assume that particles are born at hadronization according to phase space without any final state interaction. In multiparticle production processes, single-inclusive (e.g. $p+p$ ), invariant particle spectra are typically exponential in $p_{\mathrm{T}}$ [35]. The exponential behavior does not require final state interactions and it can be due to phase space population at hadronization. The slope parameter in $p+p$ collisions are independent of the particle species [36]. At RHIC, the $\rho^{0}$ spectra in minimum bias and high multiplicity $p+p$ are exponential in $p_{\mathrm{T}}$ up to $1.1 \mathrm{GeV} / c^{2}$ with slope parameters of $\sim 180 \mathrm{MeV}$ [37]. These results are also independent of the multiplicity.

However, the $\rho^{0}$ mass measured in $p+p$ at RHIC is multiplicity dependent (the mass shift in high multiplicity is higher than in minimum bias $p+p$ collisions) [16], which is opposite to the slope parameter that is multiplicity independent. Note that the systematic errors are correlate among themselves and between the two measurements. We will demonstrate that the mass should be independent of $p_{\mathrm{T}}$ as well as multiplicity at hadronization without final state interactions. According to quantum mechanics, a resonance at rest is described by a probability amplitude that is proportional to a non-relativistic Breit-Wigner distribution. When the energy conservation law is imposed in the partition of string fragmentation into multiple hadrons, a phase space factor, similar to the Boltzmann factor in thermal distribution, has to be added to the nonrelativistic Breit-Wigner distribution

$$
P\left(M, p_{\mathrm{T}}\right) \propto \frac{\Gamma / 2}{\left(M-M_{0}\right)^{2}+\frac{\Gamma^{2}}{4}} \times \exp \left(\frac{-m_{\mathrm{T}}}{T}\right) .
$$

where $M$ is the invariant mass $(M), \exp \left(\frac{-m_{\mathrm{T}}}{T}\right)$ is the phase space, $m_{\mathrm{T}}$ equals $\sqrt{M^{2}+p_{\mathrm{T}}^{2}}$ and $\mathrm{T}$ equals $160 \mathrm{MeV}$ [37]. However; as discussed previously, the phase space does not describe the mass shift of the $\rho$ meson measured at RHIC, CERN, and LEP.

Since the phase space of a non interacting multiparticle state cannot explain the distortion of the $\rho^{0}$ line shape, we can conclude that the phase space in $p+p$ collisions also accounts for hadrons scattering and forming resonances. In the case of the $\rho^{0}, \pi^{+} \pi^{-} \rightarrow \rho^{0} \rightarrow \pi^{+} \pi^{-}$. This can be pictured in the string fragmentation particle production scenario, where the string breaks several times, two pions are formed, they scatter, and form a $\rho^{0}$. Such interactions are significant and modify the $\rho^{0}$ spectral shape in $e^{+} e^{-}$and $p+p$ from a relativistic $p$-wave Breit-Wigner function.

Using the $p+p$ data measured by STAR [16] and the rescattering formalism of [39], we can test the ideas of a mass shift having a $\pi^{+} \pi^{-}$scattering component. The di-pion production is given by Eq. 21 of [39]. For the $\rho^{0}$ which is p-wave $\ell=1$ Eq. 21 becomes

$$
|T|^{2}=|D|^{2} \frac{\sin ^{2}\left(\delta_{1}\right)}{\mathrm{PS}^{\prime}}+\frac{|A|^{2}}{\mathrm{PS}^{\prime}}\left|\alpha \sin \left(\delta_{1}\right)+\mathrm{PS}^{\prime} \cos \left(\delta_{1}\right)\right|^{2}
$$

The $\delta_{1}$ is the p-wave phase shift $\left(\rho^{0}\right)$ and $\frac{\sin ^{2}\left(\delta_{1}\right)}{\mathrm{PS}^{\prime}}$ in our case becomes the Breit-Wigner times phase space equation 1. $|D|^{2}$ becomes the direct production of $\rho^{0}$ for the $p_{\mathrm{T}}$ range 600 to $800 \mathrm{MeV} / \mathrm{c} .|A|^{2}$ is the phase space overlap of di-pions in the $\ell=1$ partial waves. The pions emerge from a close encounter in a defined quantum state with a random phase. The 


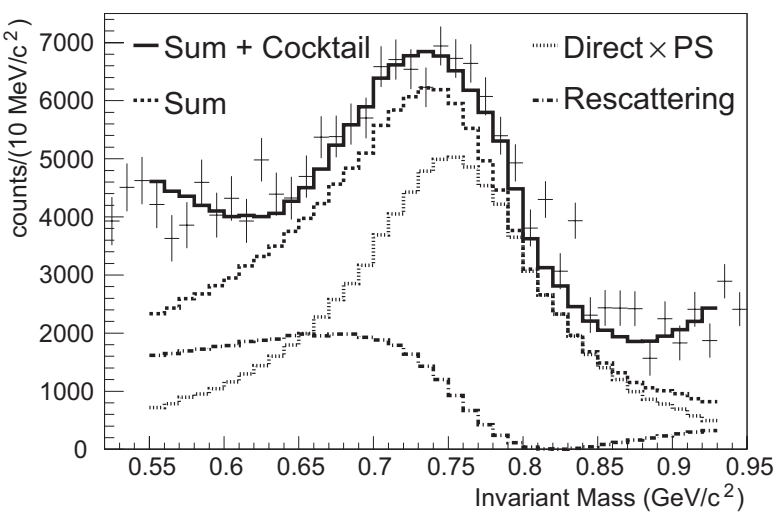

FIG. 7: Fit to the data using equation 2 (solid line), the direct $\rho^{0}$ contribution term times the phase space (dashed line), the rescattering term (dash-dotted line) and the sum of the both (dashed line).

emerging pions can re-interact or re-scatter through the pwave quantum state or a phase shift. The phase space overlap comes from sampling the $\pi$ spectrum from $p+p$ collisions, where the sum of the $\pi^{+} \pi^{-}$has the correct $p_{\mathrm{T}}$ range. The dipion mass spectrum for this $p_{\mathrm{T}}$ range falls with increasing dipion invariant mass. The variable $\alpha$ is related to the real part of the di-pion rescattering diagram and measures the range of interaction [39].

In order the fit the $p+p$ data we need to add the rest of the cocktail of resonances used by STAR [16]. Such a fit is shown in Fig. 7 (solid line), where the direct $\rho^{0}$ term time the phase space (dotted line) and the rescattered term (dash-dotted line) plus the sum (dashed line) are also depicted.

STAR measured the $\rho^{0}$ at midrapidity and NA27 measured at the forward region. In addition, there is the energy difference of $\sqrt{s_{\mathrm{NN}}}=200$ and $27.5 \mathrm{GeV}$, respectively. All these facts are consistent our model, the difference between the $\rho^{0}$ mass shift measure by STAR and NA27 is due to pion multiplicity. Similar arguments is valid for the $\rho$ measurements at CERN-LEP at $\sqrt{s_{\mathrm{NN}}}=90 \mathrm{GeV}$ indicating that there is also significant scattering interactions in $e^{+} e^{-}$reactions.

\section{CONCLUSIONS}

There are various evidences for equilibration of the matter formed at $\mathrm{Au}+\mathrm{Au}$ collisions at $\sqrt{s_{\mathrm{NN}}}=200 \mathrm{GeV}$ (e.g. $v_{2}$ measurements, relative particle yields described by thermal models, $\gamma_{S}=1$, etc). All these together with the constituent quark scaling are strong signatures of sQGP. I also discussed that the natural mass of the $\rho$ meson should be measured in exclusive reactions only. I showed that a shift on the position of the $\rho^{0}$ peak has been measured and that the phase space does not account for the $\rho(770)^{0}$ mass shift measured at RHIC, CERNLEBC-EHS, and CERN-LEP. In addition, we discussed the phase space in $p+p$ collisions and concluded that there are significant scattering interactions in $p+p$ reactions.

\section{Acknowledgement}

I would like to thank F. Laue, R. Longacre, T. Ullrich, and Zhangbu $\mathrm{Xu}$ for the lively discussions.
[1] P. Huovinen, nucl-th/0305064; P. Kolb, and U. Heinz, nuclth/0305084; E. V. Shuryak, Prog. Part. Nucl. Phys. 53, 273 (2004).

[2] M. Oldenburg et al., nucl-ex/0510026.

[3] X. Cai et al., nucl-ex/0511004.

[4] J. Adams et al., Phys. Rev. C 72, 014904 (2005).

[5] X. Dong et al., Phys. Lett. B 597, 328 (2004).

[6] J. Adams et al., Phys. Rev. Lett. 92, 052302 (2004); J. Adams et al., nucl-ex/0504022.

[7] J. Dunlop et al., nucl-ex/0510073.

[8] D. Molnar and S. A. Voloshin, et al., Phys. Rev. Lett. 91, 92301 (2003).

[9] J. Adams et al., Phys. Rev. Lett. 92, 112301 (2004).

[10] J. Adams et al., nucl-ex/0308033.

[11] L. Molnar et al., nucl-ex/0507027.

[12] J. Speltz et al., nucl-ex/0512037.

[28] P. Braun-Munzinger, I. Heppe, and J. Stachel Phys. Lett. B, 465, 15 (1999); N. Xu and M. Kaneta, Nucl. Phys. A 698, 306 (2002).

[14] E. Schnedermann, J.Sollfrank, and U. W. Heinz Phys. Rev. C, 48, 2462 (1993); U. A. Wiedemann and U. W. Heinz, Phys. Rev. C 56, 3265 (1997).

[34] J. Adams et al., Phys. Rev. C, 71, 064902 (2005);

[16] J. Adams et al., Phys. Rev. Lett. 92, 092301 (2004).

[17] G. Agakishiev et al., Phys. Rev. Lett. 75, 1272 (1995); B. Lenkeit et al., Nucl. Phys. A 661, 23 (1999).
[18] R. Arnaldi et al., Phys. Rev. Lett. 96, 162302 (2006);

[19] R. Rapp and J. Wambach, Adv. Nucl. Phys. 25, 1 (2000).

[20] M. Aguilar-Benitez et al., Z. Phys. C 50, 405 (1991).

[21] P.D. Acton et al., Z. Phys. C 56, 521 (1992).

[22] G.D. Lafferty, Z. Phys. C 60, 659 (1993); (private communication).

[23] K. Ackerstaff et al., Eur. Phys. J. C 5, 411 (1998).

[24] D. Buskulic et al., Z. Phys. C 69, 379 (1996).

[25] P. Abreu et al., Phys. Lett. B 298, 236 (1993).

[26] S. Eidelman et al., Phys. Lett. B 592, 1 (2004).

[27] H.W. Barz et al., Phys. Lett. B 265, 219 (1991).

[28] P. Braun-Munzinger (private communication).

[29] P.F. Kolb and M. Prakash, nucl-th/0301007.

[30] R. Rapp, Nucl. Phys. A 725, 254 (2003).

[31] W. Broniowski et al., Phys. Rev. C 68, 034911 (2003).

[32] S. Pratt and W. Bauer, Phys. Rev. C 68, 064905 (2003).

[33] P. Granet et al., Nucl. Phys. B 140, 389 (1978).

[34] J. Adams et al., Phys. Rev. C 71, 064902 (2005).

[35] R. Hagedorn, Relativistic Kinematics, W.A. Benjamin, 1963; E. Byckling and K. Kajantie, Particle Kinematics, Wiley, 1973.

[36] I.G. Bearden et al., Phys. Rev. Lett. 78, 2080 (1997).

[37] P.Fachini et al., hep/0608029 (2006).

[38] J. Adams et al., Phys. Rev. Lett. 92, 112301 (2004). Addison Wesley, 1985.

[39] Ron S. Longacre, nucl-th/0303068 (2003). 